\title{
Permanent Magnet Linear Synchronous Generator for an Oscillating Hydrofoil in a Tidal Current Regime
}

\author{
S. Tarafdar, M. Abroshan, and M. Mirsalim, Senior Member, IEEE
}

\begin{abstract}
This paper presents a novel method to extract energy from tidal currents by using a permanent magnet linear synchronous generator for an oscillating hydrofoil. In this work, an initial design of a linear generator is proposed for a biomimetically inspired oscillating hydrofoil system as a tidal energy generator. The device is designed to manipulate the flow stream and contained energy. It demonstrates a noticeable increase in efficiency when compared with data from existing industrial prototypes. Thus, a heightened theoretical coefficient of power and decreased cycle times are calculated for the device. The whole system is surveyed from the hydrofoil to the sample load. The main aim of the paper is to depict a new design in handling the hydrofoils in a tidal current regime.
\end{abstract}

Index Terms-Battery, oscillating hydrofoil, permanent magnet linear synchronous generator, rectifier, tidal currents.

\section{INTRODUCTION}

NCREASING demand for energy and the decreasing supplies of fossil fuels, water current and wind are emerging as alternative sources of renewable energy. The global energy requirements are primarily provided by the combustion of fossil fuels. In 2007, the global share of energy from fossil fuels was $88 \%$ of the total primary energy consumption [1].The consequence of this heavy dependence on fossil fuels is becoming increasingly concerning. These fuels have limited potential and, at the current rate of exploitation, it is expected that these resources will deplete within the coming decades [2,3]. Renewable energy technologies are becoming an increasingly favorable alternative to conventional energy sources to assuage these carbon-based fuels related issues.

Tidal energy offers a vast and reliable energy source, currently, the harnessing of tidal energy from the rise and fall of the tides has been exploited on a commercial scale using tidal barrage systems [4,5]. Recent efforts to exploit this predictable energy source have been directed towards the kinetic energy in tidal currents [6]. The extractable potential in the UK has been reported to be 22 TWh per year and 17 TWh per year for the rest of Europe [7]. Other reports give estimates of $48 \mathrm{TWh}$ per year for Europe [8] and as high as 58 TWh per year in the UK alone [9] (note that these are estimates for tidal current potential only). Much of the extractable energy is to be found in sites of depths below $40 \mathrm{~m}$.

Manuscript received May 29, 2012; revised January 15, 2013.

S. Tarafdar is with the Amirkabir University of Technology, Center of Excellence in Power Systems, Tehran, Iran (e-mail: tarafdar61@aut.ac.ir).

M. Abroshan is with the Islamic Azad University, Shahre Rey Branch, Tehran, Iran (e-mail: abroshan@aut.ac.ir).

M. Mirsalim is with the Amirkabir University of Technology, Center of Excellence in Power Systems, Tehran, Iran (e-mail: mirsalim@aut.ac.ir).
In recent years, a new type of flow energy recovery paradigm based upon a novel flapping-foil technology has been proposed [10]. A hydrofoil prototype named Stingray was developed and tested which resulted in a $150 \mathrm{kw}$ hydraulic power [11]. Hydraulic system with many moving parts is required to actuate and smooth high pressure thrusts. This is an energy transfer, with inherent losses in efficiency, internal viscous friction, mechanical friction, thermal losses and finally electrical losses. This system can be costly and add considerable weight to any device where deployed. The working medium of oil in a marine environment is also cause for concern [12]. As mentioned before, Engineering Business Ltd developed the "Stingray" oscillating hydrofoil as a developmental prototype to prove the robustness and feasibility of the concept, the design and technology [13]. As it has proved the inflated development costs of this device in the current economic climate is prohibitive and has caused the project to be suspended [14]. The model outlined here has similar principles of operation to that of "Stingray" however the model described here is using a different power take-off system, especially that a PMLSG has been used in place of a hydraulic power take-off system which is a novel design. This removes the inherent inefficiencies and complexities of hydraulic power take-off systems.

Linear generator for power take-off system would be very beneficial to increase of the efficiency and simplicity of the design because of their direct connection to the mechanical part. In oscillating wave point absorbers, linear generators have been found to be the superior power take off choice [15].

Linear synchronous devices have been shown to be more favorable and reliable, with efficiencies of $90 \%$ compared with $82 \%$ of comparable induction devices [16].

In this paper a hydrofoil has been surveyed which drives a permanent magnet linear synchronous generator (PMLSG) and this generator is connected to a sample load through a rectifier in combination with a battery that its output is gone through an inverter. As the dimensions of the hydrofoil are quite considerable, a special PMLSG should be designed to be compatible with the hydrofoil. The results of the work that was done for AWS generator design is used which is very suitable for this particular design [17]. The load power profile and the battery profile are shown at the end of this paper for a definite period of time as the main results of this work which should be considered carefully.

\section{MODELING OF HYDROFOIL}

The main principal of operation is relatively quite simple. Given a positive or negative angle of attack relative to the 
tidal current, the hydrofoil will rise and fall in an oscillating motion. The rate at which this cycle occurs is dependent on a considerable number of hydrodynamic and mechanical variables. The instantaneous power, $P$, available to an oscillating hydrofoil is given by $p=1 / 2 \rho \mathrm{A} v^{3}$ where $\rho$ is the fluid density, A the planer foil area and $\mathrm{V}$ the velocity of the fluid stream.

The lift force that drives the device is dependant on the flow velocity and density, the foil surface area and the foil profile characteristics, namely it's lift and drag coefficients for an optimum angle of attack. Unlike conventional rotational technologies which are used to generate at constant rotational speed, the lift acting on the oscillating hydrofoil approximates a sinusoidal decay from the horizontal to vertical arm positions unless the foil is adjusted by a specified control system to operate at the optimum angle of attack so that the system works in the best condition and the lift force does not form a sinusoidal shape. This non linear velocity and loss in momentum cause the device to inherently have a large degree of mechanical complexity in developing and optimizing the power output. As the lift generation reduces in certain oscillation angles, the degree of oscillation has to be limited (approx $35^{\circ}$ ) to prevent significant loss of lift. The maximum lift is governed by empirical optimum angle of attack. This angle of attack is relative to the tidal current velocity, and as the hydrofoil is continually in motion through the tidal current, this angle of attack must be controlled and dynamically optimized to maintain efficient performance and operation. Fig. 1 shows an oscillating hydrofoil structure.

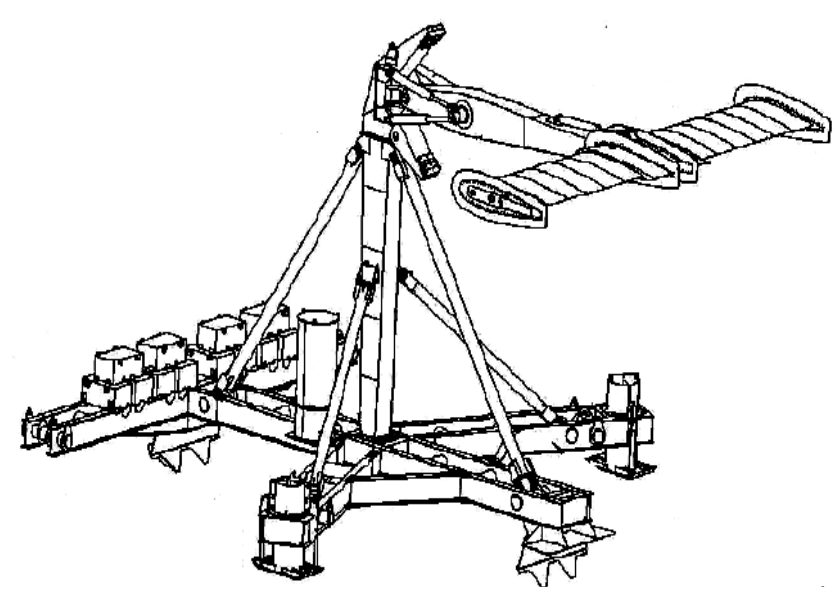

Fig. 1. Machine structure of an oscillating hydrofoil in a tidal current [15]

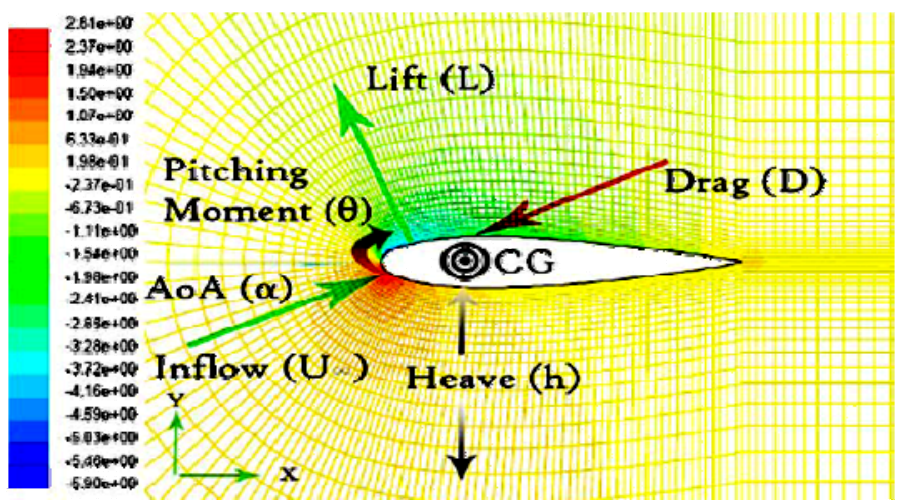

Fig. 2. Machine structure of an oscillating hydrofoil in a tidal current [15]
Control mechanism is done by program logic control (PLC), which continually monitors mechanism parameters, the tidal current velocity and the arm position. The PLC signal then controls a hydraulic ram mounted on the oscillating arm to adjust the hydrofoil angle of attack. Holding the hydrofoil stably at its optimum angle of attack is necessary, if the foil tends to wobble it significantly reduces lift, increases drag and a serious problem arises.

Furthermore this same system is used to overcome the pitching moment on the hydrofoil, stop its motion and return the cycle in the opposite direction. Empirical and mathematical models characterizing their locomotion and maneuverability are based on pitching moment and heaving movements of symmetrical hydrofoils, which concurrently heave and rotate during their cycle. In this paper, this assumption has been made that a PLC system acts which keeps the angle of attack at a certain range and changes the direction of the oscillation. Hydrofoil equations of motion are defined by eqn(1) and eqn(2).

$$
\begin{gathered}
h(t)=h_{o} \sin (\omega t) \\
\theta(t)=\theta_{o} \sin (\omega t+\psi)
\end{gathered}
$$

where $h_{0}$ is the heave amplitude, $\omega$ is the cycle frequency $($ rad.s-1), $t$ is time (s), $\theta$ is the pitch angle and $\psi$ is the phase angle (rad) between pitch and heave. The resultant angle of attack is described by;

$$
\alpha(t)=-\arctan \left[\frac{h(t)}{U_{\infty}}\right]+\theta(t)
$$

Where $\alpha$ is the angle of attack (AoA) and $U_{\infty}$ is the incident flow velocity. A hydrofoil profile is characterized by its lift, drag and pitching moment, for a range of angle of attack. Most common hydrofoil profiles, especially symmetrical foils as used in oscillatory processes, are well understood for steady state flow conditions. Their characteristics are depicted in terms of $\mathrm{Cl} \& \mathrm{Cd}$; the lift and drag coefficients for NACA0015 are shown in Fig. 4 and Fig. 5 respectively.

The lift and drag coefficients are used to calculate lift and drag forces. They are as follows:

$$
\begin{aligned}
& L=\frac{1}{2} \rho S C_{/} U_{\infty}^{2} \\
& D=\frac{1}{2} \rho S C_{d} U_{\infty}^{2}
\end{aligned}
$$

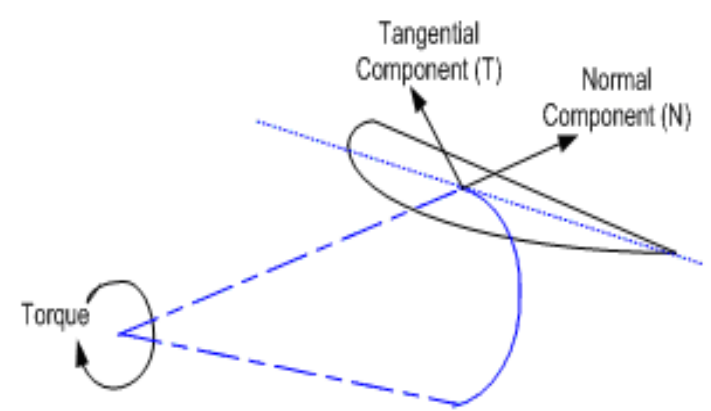

Fig. 3. Resultant tangential force on a hydrofoil 


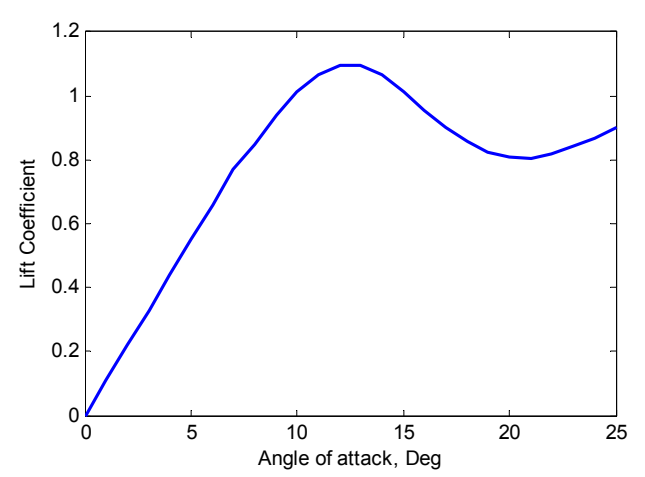

Fig. 4. Coefficient of lift in a $2 \mathrm{~m} / \mathrm{s}$ tidal current.

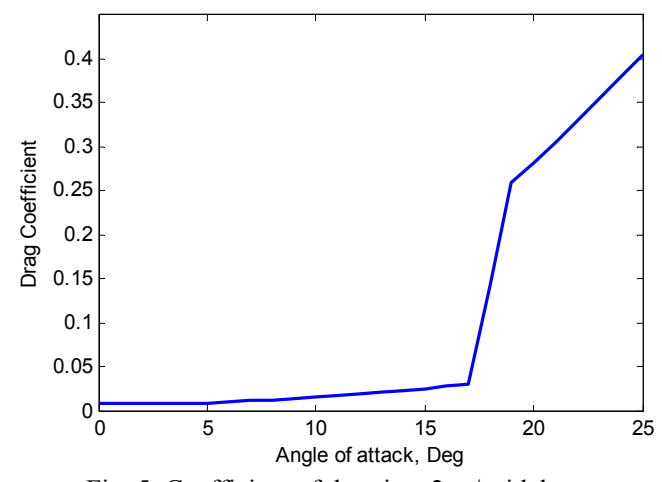

Fig. 5. Coefficient of drag in a $2 \mathrm{~m} / \mathrm{s}$ tidal current

The tangential force $T$ that is the effective force for a given angle of oscillation $\beta$ is shown in Fig. 3 and calculated by Eq. (6);

$$
T=L \cos \beta+D \sin \beta
$$

It should be noted that the drag experienced on the foil, can have positive effects depending on the phase of the cycle. Further more induced drag and stall at extreme angles of rotation can be used to accelerate change in oscillation direction and decrease the cycle time. The torque applied around the centre of rotation is dependant on the arm length or the radius of rotation. The instantaneous torque experienced is a product of the resultant Tangential force above by the Arm length as shown by Eq. (7).

$$
\text { Torque }=T \times L
$$

The cyclic torque is resolved by calculating the resultant tangential forces through the cycle angle of rotation from $\beta_{\text {min }}$ to $\beta_{\max }$. The output power cycle can then be calculated for a specified cycle time $\mathrm{C}_{\mathrm{t}}$ by Eq. (9).

$$
\begin{aligned}
& \omega=\left(\frac{\left(\frac{4 \pi \beta_{\max }}{360}\right)}{C_{t}}\right) \\
& P=\operatorname{Torque} \times[\omega \sin (\phi)]
\end{aligned}
$$

where $\omega$ is the angular velocity in radians per second, and $\varphi$ is the cycle phase angle. This way the power can be calculated. This assumption has been made that a tidal current with a $2 \mathrm{~m} / \mathrm{s}$ flow velocity exists which lasts for a period of time, a cycle time of 70 seconds, a NACA0015 hydrofoil area of $45 \mathrm{~m}^{2}$, arm length of 10.9 meters and operates with a power coefficient $\left(\mathrm{C}_{\mathrm{P}}\right)$ of 0.12 .

\section{MODELLING OF PMLSG}

As the dimensions of the hydrofoil are quite considerable, a special PMLSG should be designed to be compatible with the hydrofoil. The results of the work that was done for AWS design has been used which is very appropriate for the new design. A PMLSG with magnets on the translator (the moving part) was chosen because:

- It has a rather high force density;

- It has a reasonable efficiency at low speeds;

- Magnets are not that expensive anymore;

- There is no electrical contact to the translator.

Fig. 6 depicts a cross-section of the generator.

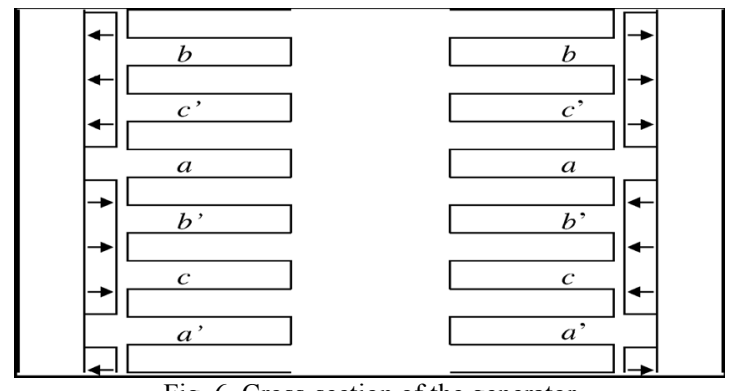

Fig. 6. Cross-section of the generator

The generator that has been used in this study is a Vernier Hybrid machine with moving secondary and surface mount permanent magnet (PM) which is presented in Fig. 6. The parameters of the PMLSG are shown in Table $I$ in the appendix. The machine model of a PMLSG can be described in rotor reference frame as follows [18].

TABLE I: PARAMETERS OF VERNIER PMLSG

\begin{tabular}{lcc}
\hline \hline Number of pole pairs & $P_{n}$ & 2 \\
\hline d-axis inductance & $L_{d}$ & $31 \mathrm{mH}$ \\
\hline q-axis inductance & $L_{q}$ & $29.5 \mathrm{mH}$ \\
\hline Stator resistance & $R$ & $\Omega 0.29$ \\
\hline Magnetic flux-linkage & $\psi_{f}$ & $0.072 \mathrm{~Wb}$ \\
\hline Mover mass & $M$ & $150 \mathrm{Kg}$ \\
\hline Pole pitch & $\tau$ & $0.1 \mathrm{~m}$ \\
\hline Friction coefficient & $B$ & $0.01 \mathrm{~N} . \mathrm{s} / \mathrm{m}$ \\
\hline \hline
\end{tabular}

$$
\begin{gathered}
{\left[\begin{array}{l}
u_{d} \\
u_{q}
\end{array}\right]=\left[\begin{array}{cc}
R_{s}+L_{d} \frac{d}{d t} & -P \frac{\pi}{\tau} v_{m} L_{q} \\
P \frac{\pi}{\tau} v_{m} L_{d} & R_{s}+L_{q} \frac{d}{d t}
\end{array}\right]\left[\begin{array}{l}
i_{d} \\
i_{q}
\end{array}\right]+\left[\begin{array}{c}
0 \\
P \frac{\pi}{\tau} v_{m} \psi_{f}
\end{array}\right]} \\
F_{T}=\frac{3}{2} P \frac{\pi}{\tau} \frac{1}{L_{d} L_{q}}\left|\psi_{S}\right|\left[2 \psi_{f} L_{q} \sin \delta-\right. \\
\left.\left|\psi_{S}\right|\left(L_{q}-L_{d}\right) \sin 2 \delta\right] \\
F_{\text {Hydro }}-F_{T}=M \frac{d v_{m}}{d t}+B v_{m}
\end{gathered}
$$

where, 
$P \quad$ Number of pole pairs;

$\tau$ Pole pitch;

$v_{m}$ Mover velocity;

$d_{m}$ Mover position;

$\psi_{f}$ Permanent magnet flux linkage;

$\psi_{S}$ Stator flux linkage;

$R_{S}$ Phase winding resistance;

$u_{d}, u_{q} \mathrm{~d}$ - and q-axis voltages;

$i_{d}, i_{q} \mathrm{~d}$ - and q-axis currents;

$L_{d}, L_{q} \quad \mathrm{~d}$ - and q-axis inductances;

$\delta$ Load angle;

$M$ Mover mass;

$B$ Friction factor;

$F_{\text {Hydro }}$ Hydrofoil arm force;

$F_{T} \quad$ Electrical thrust force;

\section{SYSTEM STRUCTURE}

The sample hydrofoil is connected to a PMLSG and a rectifier is used to transfer the resultant power to a battery to charge it. The battery is connected to a SPWM inverter to produce a sinusoidal voltage which is suitable for the sample load. In Fig. 7 the block diagram shows the whole system. As mentioned before, tidal currents (a horizontal motion) are a result of the rise and fall of the water level due to tides (a vertical motion). The effects of tidal currents on the movement of water in and out of bays and harbors can be substantial, in fact the tidal current profile forms a sinusoidal shape with a cycle time of 12 hours and 25 minutes [18]. Because of this quite long time the simulation is done during a specified device cycle time for a $2 \mathrm{~m} / \mathrm{s}$ tidal current velocity. The translator of the PMLSG moves with the heave speed of the hydrofoil so the speed is used as the relevant input into the generator. The way that the battery is charged and discharged is of great significance and should be carefully noted. For instance in high velocities of tidal current the battery is charged and the load provided and in low velocities of tidal current which the device is not capable of providing the load energy, the battery does its role to support the load. A transformer has been inserted to regulate the voltage and eliminate the harmonics.

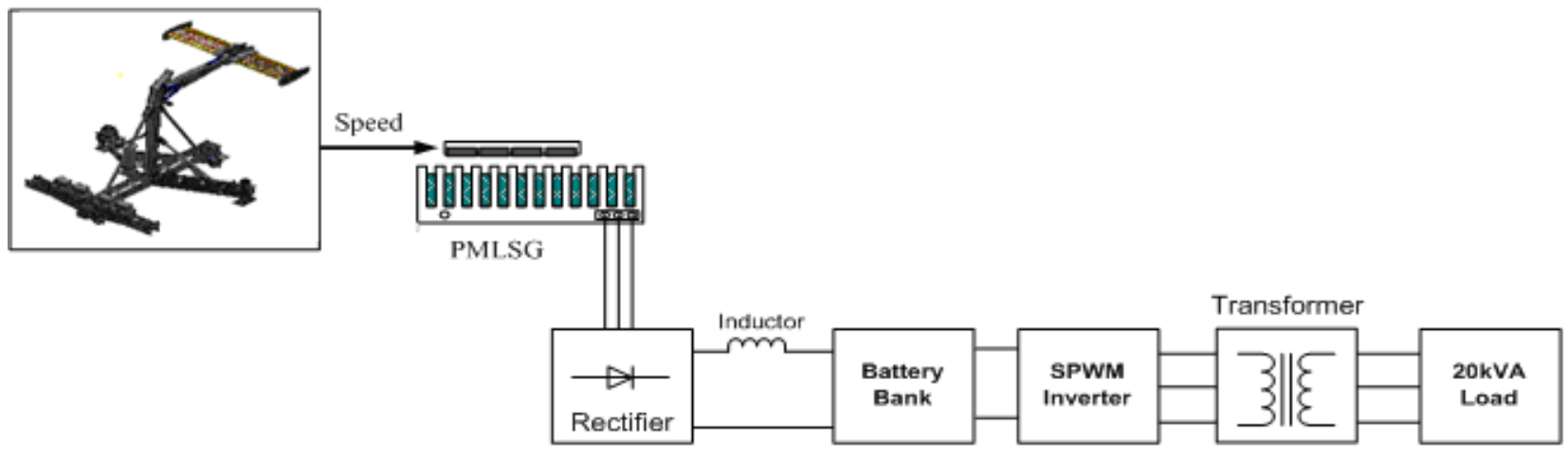

Fig. 7. Block diagram of the Hydrofoil-PMLSG system

Battery bank with nominal voltage as equal to $\mathrm{Cn}(\mathrm{Ah})$ is allowed to be discharged to a limited amount and this will be determined by designer. Regarding to PMLSG output power and demanded load, the battery SOC (State of Charge) along simulation time will be derived as follows:

$$
C(t)=C(t-1)+n_{B} \frac{P_{B}(t)}{V_{B U S}} \Delta t
$$

where:

$C(t)=$ accessible battery capacity (Ah) at time t;

$n_{B}=80 \%=$ charge round-trip efficiency

$n_{B}=100 \%=$ discharge round-trip efficiency

$V_{B U S}=\mathrm{DC}$ bus voltage

$P_{B}(t)=$ battery discharge input/output power

$\Delta \mathrm{t}=$ time step of simulation

\section{SimULATION}

Because of the long cycle time of the tidal current, the simulation has been done for a specified tidal current velocity. The customary velocity of $2 \mathrm{~m} / \mathrm{s}$ is chosen. It should be noted that the results are obtained for a certain cycle time of the device that depends on the control system and hydrodynamic parameters. It has been assumed that $\mathrm{Cl} \& \mathrm{Cd}$ follow the patterns that are shown in Fig. 4 and Fig. 5 and it is postulated that the angle of attack is kept in a certain range and not only at its optimum. The sample hydrofoil is connected. Fig. (8-a) depicts the generator speed for a specified cycle time. Fig. (8-b) shows the hydrofoil torque for a complete oscillation movement in a cycle time. The output power of the rectifier is presented in Fig. (8-c). Fig. (8-d) is indicative of hydrofoil mechanical power. The inverter voltage and the load voltage are exhibited in Fig. (8-e) and Fig. (8-f) respectively. Fig. (8-g) and Fig. (8-h) describes the load power profile and the battery energy profile for a definite period of time. The battery energy profile shows the charge and discharge of the battery during a certain time. 


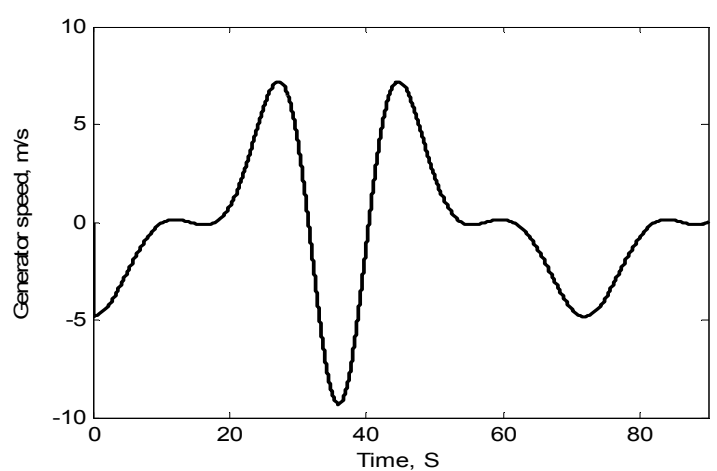

(a)

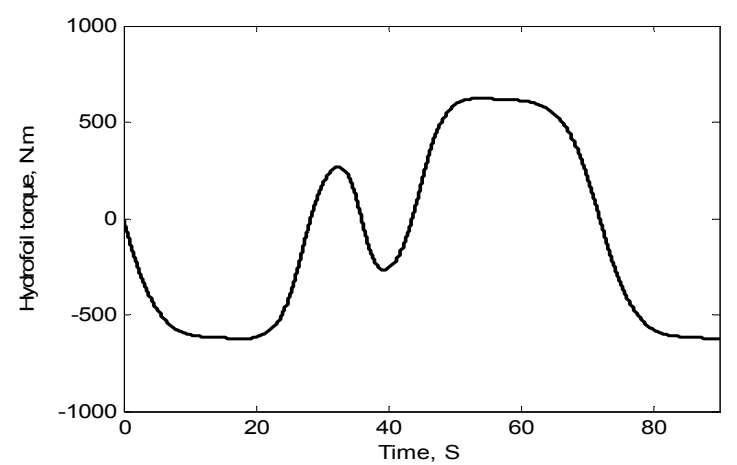

(b)

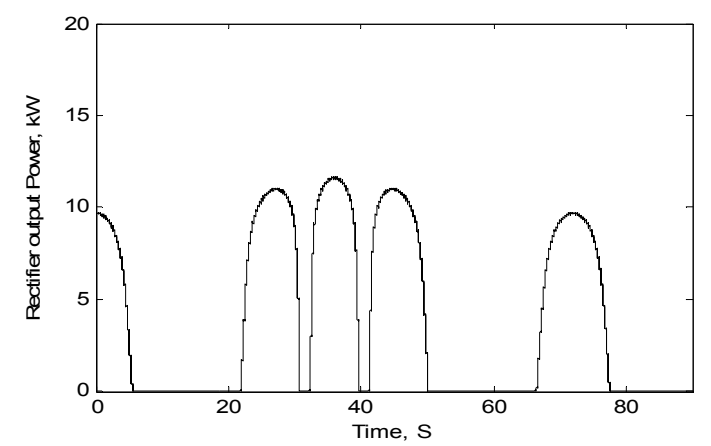

(c)

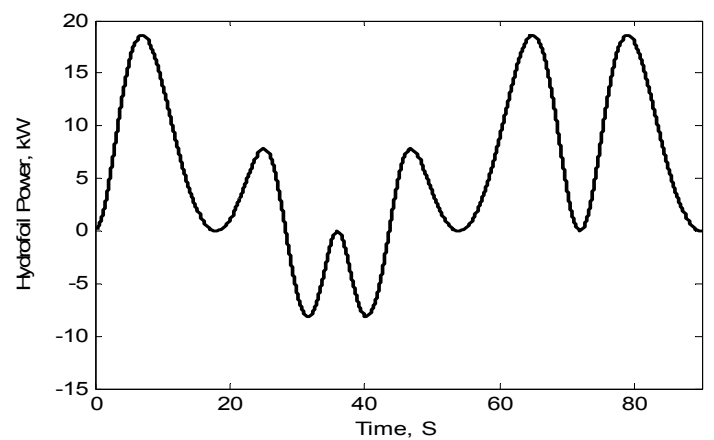

(d)

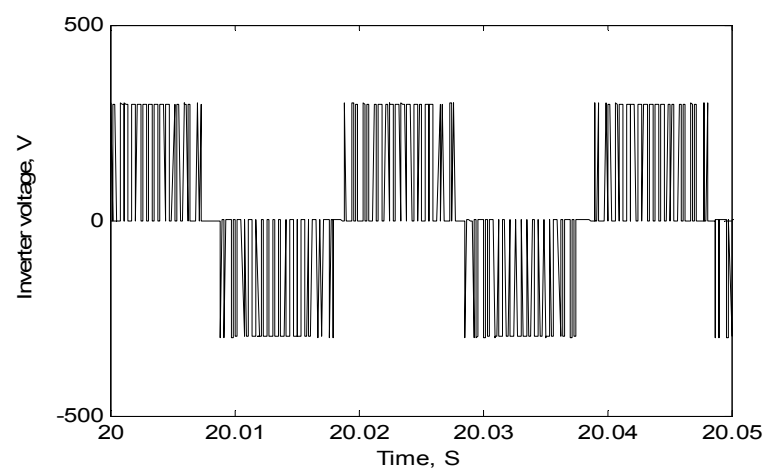

(e)

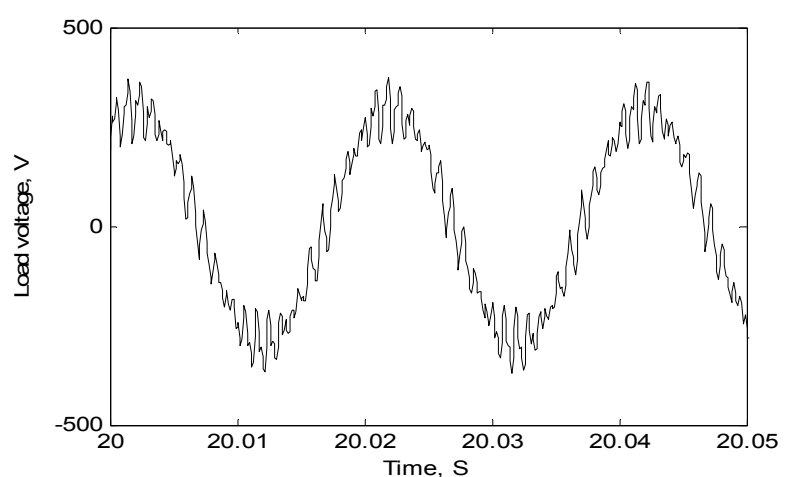

(f)

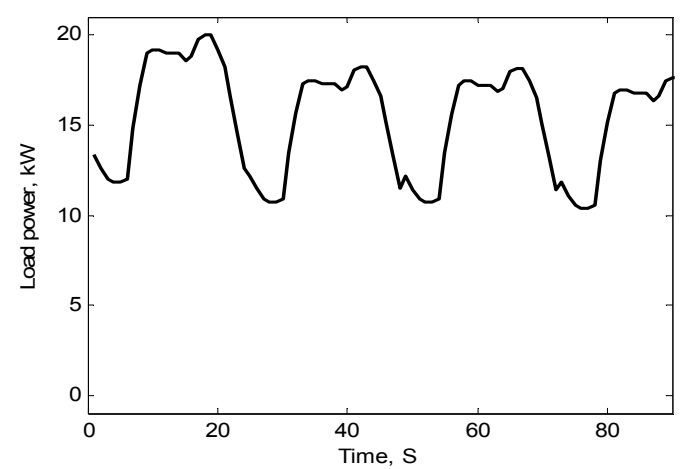

(g)

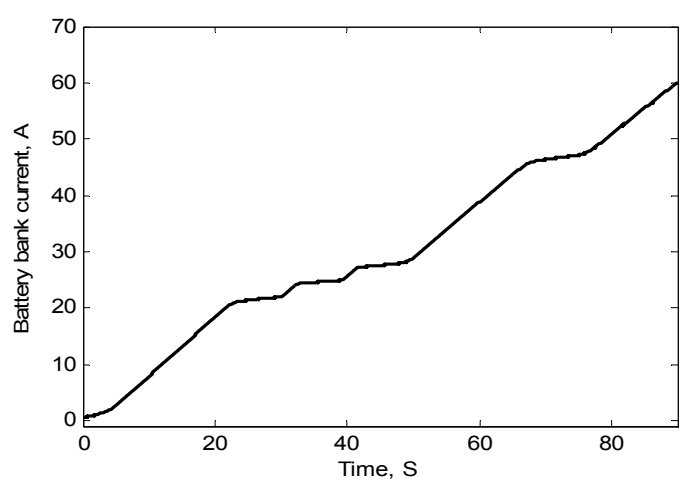

(h)

Fig. 8. Simulated responses of the Hydrofoil power system with PMLS generator: (a) generator velocity, (b) Hydrofoil force, (c) rectifier output power, (d) rectifier output current, (e) inverter output voltage, (f) load voltage, (g) load power profile and (h) battery current profile.

\section{CONCLUSION}

A system comprising of a hydrofoil, a permanent magnet linear synchronous generator, rectifier, a battery, inverter and a sample load has been surveyed under a certain condition of the tidal current. Under specific circumstances this can be used to extract energy from tidal currents so that a sample loads such as a typical IEEE load can be provided during a 24 hours. It should be noted that this is a novel method of extracting energy and can be used for local loads in remote areas near suitable shores which have strong tidal currents.

\section{REFERENCES}

[1] N. Tanaka, World Energy Outlook 2007, France: OECD, 2007.

[2] N. R. E. Laboratory, Technology options for the near and long term: U.S. Climate Change Technology Program, 2005. 
[3] S. Carley, "State renewable energy electricity policies: an empirical evaluation of effectiveness", Energy Policy, vol. 37, No. 8, Elsevier, 2009, pp.3071-81.

[4] K. V. Alphen, H. S. Kunz, and M. P. Hekkert, "Policy measures to promote the widespread utilization of renewable energy technologies for electricity generation in the Maldives," Renewable and Sustainable Energy Reviews, vol. 12, No. 7, Elsevier, 2008, pp.1959-1973.

[5] P. R. Cave and E.M. Evans, "Tidal stream energy systems for isolated communities," Alternative Energy Systems-Electrical Integration and Utilisation, Pergamon Press, 1984.

[6] M. Watchorn, T. Trapp, and A. A. M. Sayigh, "Tidal stream renewable offshore power generation (TS-Ropg)," in World Renewable Energy Congress VI, Pergamon, 2000, pp.2664-67.

[7] Black \& Veatch Consulting, Ltd., "Tidal stream resource report," Peer Review, Issue 1, 2004.

[8] Non-nuclear energy - JOULE II project: The exploitation of tidal and marine current, wave energy - project results, IT Power Ltd, European commission press, 1996.

[9] Tidal Stream Energy Review, Department of Trade and Industry, UK, 1993.

[10] W. McKinney and J. DeLaurier, "The Wingmill: an oscillating-wing windmill," Energy, No. 2, 1981, pp.109-115.

[11] M. Campbell, "Concept development of an oscillating tidal power generator," in Proc. of ASME Fluids Engineering Division Conf., vol. 2, 2002, pp.1065-1074.

[12] N. J. Baker, "Linear Generators for Direct Drive Marine Renewable Energy Converters," PhD dissertation, Dept. Engineering, University of Durham, UK, 2003.

[13] Research and development of a 150kw tidal stream generator - phase 1, Department of Trade and Industry, The Engineering Business Ltd, 2002.

[14] Research and development of a 150kw tidal stream generator - phase 2, Department of Trade and Industry, The Engineering Business Ltd, 2003.

[15] J. Glynn, "Design of Biomimetic Passive Control for Optimization of Oscillating Hydrofoils in Tidal Energy Capture," Msc Thesis, Dept. of Mechanical Engineering, University of Strathclyde, UK, 2006.

[16] J. Wang, G. W. Jewell, and D. Howe, "A general framework for the analysis and design of tubular linear permanent magnet machines," IEEE Transactions on Magnetics, vol. 35, No. 3, IEEE, 1999, pp.1986-2000.

[17] H. Polinder, M. E. C. Damen, and F. Gardner, "Linear PM generator system for wave energy conversion in the AWS," IEEE Transactions on Energy Conversion, vol. 19, No. 3, IEEE, 2004, pp.583-589.

[18] J. F. Gieras and Z. J. Piech, Linear Synchronous Motors: Transportation and Automation Systems, CRC Press, 1999.

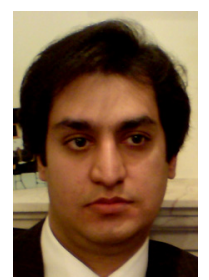

Soroush Tarafdar was born in Tehran, Iran, on 1982 He received his B.Sc. degree in Electerical Engineering from K.N.Toosi University of Technology (KNTU), Tehran, Iran in 2006 and Master of Science degree in Electrical Engineering in field of energy management from Amirkabir University of Technology (Tehran Polytechnic), Tehran, Iran in 2010. His research interest includes Renewable energies, Power Electronics, DGs and their connection to the grid, Power Quality Analysis, Reliability and Cost Assessment and Energy storage systems.

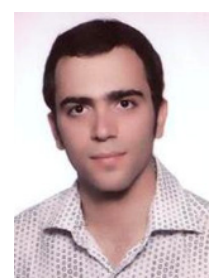

Mohammad Abroshan was born in Tehran, Iran, on September 21, 1983. He received the B.Sc. degree in electrical engineering from Tabriz University of Tarbiat Moallem, Tabriz, Iran, in 2006 and the M.Sc. degree from the Amirkabir University of Technology (Tehran Polytechnic Uni.), Tehran, Iran, in 2009.

His research interests cover many aspects of Power Electronics, Renewable energies, Variable speed Drives, Wind Energy, Optimization algorithms, Intelligent Controls, as well as Design and Analysis of Electrical Machines.

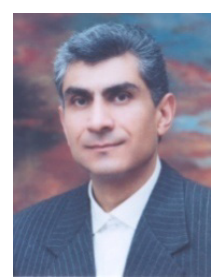

Mojtaba Mirsalim was born in Tehran, Iran, on February 14, 1956. He received the B.S. degree in EECS/NE and the M.S. degree in nuclear engineering from the University of California, Berkeley, in 1978 and 1980, respectively, and the Ph.D. degree in electrical engineering from Oregon State University, Corvallis, in 1986. Since 1987, he has been at Amirkabir University of Technology, Tehran, where he has served five years as the Vice Chairman and more than seven years as the General Director in Charge of Academic Assessments, and currently is a Full Professor in the department of Electrical Engineering where he teaches courses and conducts research in energy conversion, electrical machine design, and hybrid vehicles, among others. His special fields of interest include the design, analysis, and optimization of electric machines, FEM, renewable energy and hybrid vehicles. He is the author of more than 90 international journal and conference papers and three books on electric machinery and FEM. He is the founder and at present, the director of the Electrical Machines \& Transformers Research Laboratory. 